Cronfa - Swansea University Open Access Repository

This is an author produced version of a paper published in :

Cronfa URL for this paper:

http://cronfa.swan.ac.uk/Record/cronfa2016

http://dx.doi.org/10.1016/j.ijfatigue.2007.01.014

This article is brought to you by Swansea University. Any person downloading material is agreeing to abide by the terms of the repository licence. Authors are personally responsible for adhering to publisher restrictions or conditions. When uploading content they are required to comply with their publisher agreement and the SHERPA RoMEO database to judge whether or not it is copyright safe to add this version of the paper to this repository. http://www.swansea.ac.uk/iss/researchsupport/cronfa-support/ 


\section{A New Model for Predicting Creep/Fatigue Crack Growth Rates in Ti 6246}

P.J.Hurley* ${ }^{1}$, M.T.Whittaker ${ }^{1}$, P.Webster ${ }^{2}$, W.J.Evans ${ }^{1}$

${ }^{1}$ University of Wales, Swansea, UK, ${ }^{2}$ Rolls-Royce plc, Derby, UK

\section{Abstract}

A new model has been developed which is capable of predicting creep/fatigue crack growth rates at ambient and elevated temperatures in Ti 6246. Predictions are based on finite element analysis and strain-control testing of plain specimens. The prediction of fatigue crack growth rates for a given crack configuration and cyclic plastic zone size is assumed to be consistent with the processes leading to crack initiation in plain-specimens. Such an assumption leads to the conclusion that a similar stress-strain profile will lead to similar lives in both the plain specimens and in the cyclic plastic zone ahead of a crack in a notched specimen. Therefore, fatigue crack growth results from the accumulation of damage in the cyclic plastic zone ahead of the crack tip. Once the damage accumulated in this element of material becomes critical, the crack propagates through the damaged region into a new region of virgin material where the process of damage accumulation begins again. The creep/fatigue model is described and assessed with reference to measured fatigue crack growth rate data for Ti 6246 at $20^{\circ} \mathrm{C}$ and $500^{\circ} \mathrm{C}$.

\section{Keywords}

creep, fatigue, damage accumulation model, cyclic plastic zone, titanium 


\section{Introduction}

Damage tolerant fatigue design methods for critical rotating components such as discs and shafts in gas turbine engines are well established. These address crack propagation through the application of fracture mechanics, assuming defects are present in the material. The prediction methods have limitations, but at lower temperatures, these are conservative. It is not clear, however, that similar conservatism is valid at higher temperatures where additional failure modes due to creep and environment interact with those arising from fatigue. This is of particular concern at the present time because current performance demands are pushing the more critically stressed features at HP compressor and turbine disc rims into an operating regime where the additional failure modes due to creep and environment may interact with those arising from fatigue during critical phases of flight cycles. Clearly there is an engineering requirement to understand and model the fatigue response under these more adverse conditions.

This paper introduces a new model designed to address this uncertainty through the construction of a damage tolerant life prediction capability for conditions where fatigue and creep interact. The model is based on the idea that, during the loading/unloading cycle of a fatigue crack, local plastic strain is contained within a small volume of material ahead of the crack tip (i.e. the process zone). At ambient temperatures, fatigue crack growth occurs as a result of the damage processes occurring within this zone. At high temperatures, creep alters the local stress conditions in the process zone and can introduce additional damage events leading to enhanced crack propagation rates (Jones et al. [1]) as a result of the creep-fatigue interaction. This process does not invoke a new failure mechanism but leads simply 
to an interaction of fatigue cracks with creep damaged material as crack growth progresses into material that has already been compromised by creep cavitation. In this paper, a loosely-coupled creep fatigue model was developed with the effects of creep and fatigue calculated separately and then combined at each crack increment to provide a prediction for total crack growth rate. The details of the individual modelling methods used are given below.

\section{Modelling Approach}

\subsection{Fatigue model}

The model is based on similar concepts to previous damage accumulation models, in which non-continuous incremental crack growth is assumed to occur once a cumulative level of damage has been reached within a small element of material ahead of the crack tip, usually referred to as the process zone (Antolovich [2], Glinka [3], Kujawski et al. [4]). In the current work, the size of the process zone was assumed to be equivalent to the width of the cyclic plastic zone. It is argued that the damage accumulated in the cyclic plastic zone ahead of a fatigue crack during repeated cyclic loading is equivalent to the damage accumulated in the cross section of a plain specimen subjected to identical stress/strain conditions during strain controlled testing. In a plain specimen, the cyclic plastic zone encompasses the entire cross section so that repeated loading leads eventually to damage accumulation and crack nucleation. Therefore, fatigue crack growth is considered to occur via a series of quasi-static increments with growth occurring once a critical level of damage has been accumulated in the crack tip cyclic plastic zone. This damage is calculated based on finite element analysis of the stress/strain conditions in the cyclic plastic zone and through correlation with strain controlled damage lifing curves. By 
summing up the individual micro-increments during crack growth, a fatigue crack length versus applied cycles curve is predicted enabling stage II fatigue crack growth rates to be predicted. No a-priori knowledge of threshold data or fatigue crack growth rate characteristics is required.

The commercial finite element package Abaqus (Abaqus [5]) was employed to calculate the stress-strain conditions at the tip of a quasi-static crack. This software provided the facility for modelling nonlinear material behaviour via a kinematic hardening methodology based on the principles originally derived by Chaboche (see Lemaitre and Chaboche [6]). The method was calibrated using stabilised fullyreversed strain controlled hysteresis loops obtained from experiments described in (Hurley et al. [7]). Benchmarking against strain controlled test data confirmed the ability of the finite element method to characterise the mechanical response of the alloy at different $\mathrm{R}$ ratios (Hurley et al. [8]).

A two-dimensional finite element mesh of a square section corner-cracked specimen (Hurley et al. [8]) was constructed (half-model) using 8-noded, isoparametric, rectangular reduced integration elements (CPE8R) with standard degenerative elements used to represent the crack tip. In two-dimensional analysis, this involved degenerating eight-node rectangular elements into six node triangles by collapsing the crack tip nodes to a single point and moving the adjacent midpoint nodes to a $1 / 4$ point location, as shown in fig.1 (Abaqus [5], Pickard [9]). This method remains the most versatile approach to model the stress-strain field around a crack tip without the need to resort to extremely fine mesh configurations (Pickard [9]). Allowing the collapsed crack tip nodes to move independently simulates a combination of linear elasticity and 
perfect plasticity, which is relevant to power-law plastic hardening materials (Abaqus [5]). The element size was reduced closer to the crack tip region so that the mechanical response could be more accurately described in this region. In metals, local plastic yielding occurs in the vicinity of cracks, blunting the crack tip and effectively eliminating the strain singularity which would occur in a purely elastic material. In this study, stress and strains values at the numerical integration point closest to the quarter-point node were used for all calculations. This point was chosen because it was located closest to midpoint of the cyclic PZ, where the stressstrain response was assumed to represent average conditions ahead of the blunted crack tip.

The number of cycles, $\Delta \mathrm{N}_{\mathrm{i}}$, for fatigue crack propagation through the cyclic plastic zone (distance $\left.=\Delta \mathrm{a}_{\mathrm{i}}\right)$ was calculated by correlating with fatigue data from strain controlled testing of plain specimens. All details of the strain controlled testing experiments are described elsewhere (Hurley et al. [7]). A single failure criterion was utilised based on "Walker strain", a widely used method for collapsing data for different mean stress fatigue tests into a single $\mathrm{R}=0$ trend curve with life, fig. 2 (Hurley et al. [7], Walker [10]). The particular form of the Walker strain used in this work is given in Eqn. 1, in which exponent $\mathrm{w}$ provides a way to normalise the data with respect to R-ratio.

$$
\varepsilon_{w}=\frac{\sigma_{\max }}{E}\left\{\frac{\Delta \varepsilon E}{\sigma_{\max }}\right\}^{w}
$$

where: $\quad \sigma_{\max }$ is the maximum stress

$\Delta \varepsilon$ is the strain range,

E is Young's Modulus and 
$\mathrm{w}$ is a fitting exponent which can vary between 0 and 1.

Crack tip Walker strain values were calculated based on stress-strain calculations obtained using nonlinear finite element methods described above. In this work, Walker strain calculations were based on the von Mises equivalent stress. It is argued that the tri-axial stress state resulting from the constraint imposed by plane strain conditions present at the tip of cracks was more suitably accounted for through use of the von Mises equivalent stress rather than the axial stress component.

Once analysis of the quasi-static crack had been completed, the geometry was remeshed to simulate Mode I crack propagation through the cyclic plastic zone, the dimensions of which having been determined using equ.2, assuming plane strain conditions at the crack tip (e.g. Dowling [11]):

$$
2 r_{s o}=a_{i}=\frac{1}{3 \pi}\left(\frac{\Delta K}{2 o_{o}}\right)^{2}
$$

In this work, the cyclic yield stress, $\sigma_{0}$, was used in the calculation of the cyclic plastic zone size while value of the elastic stress intensity factor, $\mathrm{K}$, for any given crack configuration were determined using Abaqus. The entire procedure was automatically controlled by a purpose-written software programme, which controlled the re-meshing process, extraction of relevant numerical data from the Abaqus output data file and calculation of $\Delta \mathrm{N}_{\mathrm{i}}$ and the cyclic plastic zone size, $\Delta \mathrm{a}_{\mathrm{i}}$, for each crack configuration. The process of fatigue crack propagation via incremental stepwise growth is illustrated schematically in Fig.3a-d. A relationship between crack length and applied cycles was therefore developed by summing individual increments of $\Delta \mathrm{a}_{\mathrm{i}}$ and $\Delta \mathrm{N}_{\mathrm{i}}$ over the full spectrum of crack lengths considered, providing a prediction of the fatigue crack growth rate, $\mathrm{da} / \mathrm{dN}$ as a function of $\Delta \mathrm{K}$. 


\subsection{Creep Model}

When components operate under conditions where creep deformation can occur, the accumulation of strain with time significantly redistributes stress at critical stress concentration features. For pure creep, the stress redistribution is sometimes mapped through reference (Boyle et al. [12]) or 'skeletal' (Hayhurst et al. [13]) stresses which are then taken to be invariant. The stresses are calculated by suitable analytical or numerical procedures, but for the calculations to be realistic the creep properties of the material must be adequately described by a constitutive equation. In some cases only steady state creep is considered (Al-Faddagh et al. [14]) which is inadequate when primary and tertiary mechanisms are present. In particular, damage processes must be included (Hayhurst et al. [15], Othman et al. [16]) and when this is done, the stresses calculated for some geometries are not always invariant. When geometric changes occur during service, and especially when such changes are associated with the initiation and growth of fatigue cracks, additional analysis procedures are required. Recent work (Evans[17]) has indicated that, provided the material properties are well described, finite element calculations based on initial strain methods (Zienkiewicz et al. [18]) realistically quantify strain accumulation, stress redistribution, creep failure times, crack initiation sites and crack growth paths. The constitutive equation must also incorporate the effects of multi-axial stress creep (Evans et al. [19) and criteria for final failure. The modelling approach used in this work addresses the development of stress-strain conditions at the tip of a crack. 
Creep modelling has been implemented through Abacus code using a creep constitutive relationship based on internal variables (Evans [17]). The relationship has the form

$$
\dot{\bar{\varepsilon}}=\dot{\bar{\varepsilon}}_{o} f\left(\xi_{\alpha}\right) \text {. }
$$

where $\dot{\bar{\varepsilon}}_{o}$ is the creep rate for virgin material (and a function of stress and temperature). The function of the internal variables $f$ takes on the value 1 for such material but thereafter is modified by the creep process. In this alloy, creep curves exhibit a primary phase of decreasing creep rate until some minimum creep rate is observed. This is followed by a tertiary stage where the creep rate continually increases until failure. Primary creep is assumed to be due to competing dislocation work-hardening and recovery mechanisms. Tertiary creep is though to be due to general long-range structure deterioration resulting from a number of potential mechanisms. One possible form for $\mathrm{f}$ is to make it a linear function of all the internal variables, so that

$$
\dot{\bar{\varepsilon}}=\dot{\bar{\varepsilon}}_{o}\left\{1+\left(h_{1}+\ldots+h_{\alpha}+\ldots+h_{n}\right)+\left(r_{1}+\ldots r_{\beta}+\ldots r_{m}\right)+\left(w_{1}+\ldots+w_{\zeta}+\ldots w_{p}\right)\right\}
$$

where $\mathrm{h}, \mathrm{r}$ and $\mathrm{w}$ are dislocation hardening, dislocation softening and damage internal variables. For virgin materials $\mathrm{h}, \mathrm{r}$ and $\mathrm{w}$ will be zero. Work hardening is a dynamic variable and softening (recovery) a static variable, while damage variables may be of either kind but are more often dynamic (Evans [17]). Postulated forms of the evolutionary equations are then

$$
\left.\begin{array}{ll}
\dot{h}_{\alpha}=-\hat{h}_{\alpha} \dot{\bar{\varepsilon}}, & \alpha=1, n, \\
\dot{r}_{\beta}=\hat{r}_{\beta}, & \beta=1, m, \\
\dot{w}_{\zeta}=\hat{w}_{\zeta} \dot{\bar{\varepsilon}}, & \zeta=1, p,
\end{array}\right\}
$$

where $\mathrm{h} \mathrm{r}$ and $\mathrm{w}$ are positive proportionality constants which are functions of $\sigma$ and $\mathrm{T}$. 
Since the internal variables in (4) occur linearly, and since equations (5) are linear in coefficients, variables of the like nature can be summed with no loss of generality. Overall hardening, recovery and damage variables $\mathrm{H}, \mathrm{R}$ and $\mathrm{W}$ are given by

$$
H=\sum_{\alpha=1}^{n} h_{\alpha}, \quad \quad \quad \quad W=\sum_{\beta=1}^{m} r_{\beta}, \quad \sum_{\zeta=1}^{p} w_{\zeta}
$$

and their evolutionary coefficients by

$$
\hat{H}=\sum_{\alpha=1}^{n} \hat{h}_{\alpha}, \quad \hat{R}=\sum_{\beta=1}^{m} \hat{r}_{\beta}, \quad \hat{W}=\sum_{\zeta=1}^{p} \hat{w}_{\zeta}
$$

The constitutive equations now become

$$
\begin{aligned}
& \dot{\bar{\varepsilon}}=\dot{\bar{\varepsilon}}_{o}\{1+H+R+W\} \\
& \dot{H}=-\hat{H} \dot{\bar{\varepsilon}}, \\
& \dot{R}=\hat{R}, \\
& \dot{W}=\hat{W} \dot{\bar{\varepsilon}}
\end{aligned}
$$

In these equations $\hat{H}, \hat{S}$ and $\hat{W}$ are positive quantities representing hardening, softening and continuum damage rates. Thus, over limited ranges of stress, hardening is taken to be a linear process with regard to strain and softening a linear process with respect to time. At any time and any point in the creeping body, the current creep rates can be calculated provided $\dot{\bar{\varepsilon}}_{o}, H, S$ and $W$ are known and these can be calculated incrementally from values of $\hat{H}, \hat{S}$ and $\hat{W}$.

Equations (8) can be integrated at constant stress to obtain a formula for the variation of creep strain with time at constant stress and temperature. The resulting equation is: 


$$
\bar{\varepsilon}=\frac{1}{\hat{H} \dot{\bar{\varepsilon}}_{o}}\left(\dot{\bar{\varepsilon}}_{o}-\frac{\hat{R}}{\hat{H}}\right)\left(1-e^{-\hat{H} \dot{\bar{\varepsilon}}_{o} t}\right)+\frac{1}{\hat{W}}\left(e^{\frac{\hat{W} \hat{R}}{\hat{H}}}-1\right)
$$

Equation (9) is of exactly the same form as the widely used $\theta$ creep curve shape function (Evans et al. [19])

$$
\varepsilon=\theta_{1}\left(1-e^{-\theta_{2} t}\right)+\theta_{3}\left(e^{\theta_{4} t}-1\right)
$$

with the replacement of $\varepsilon$ by $\bar{\varepsilon}$ incorporating the known good fit of the equation in complex stress states. Comparison of equations (9) and (10) enables identification of the constitutive equation coefficients $\dot{\bar{\varepsilon}}_{o}, \hat{H}, \hat{R}$ and $\hat{W}$ with the $\theta$ coefficients at the same $\mathrm{T}$ and $\sigma$.

$$
\left.\begin{array}{l}
\dot{\bar{\varepsilon}}_{o} \equiv \theta_{1} \theta_{2}+\theta_{3} \theta_{4} \\
\dot{H} \equiv \theta_{2} / \dot{\bar{\varepsilon}}_{o} \\
\dot{R} \equiv \theta_{2} \theta_{3} \theta_{4} / \dot{\bar{\varepsilon}}_{o} \\
\dot{W} \equiv 1 / \theta_{3}
\end{array}\right\}
$$

The techniques for determining the quantities $\theta_{1}, \theta_{2}, \theta_{3}$ and $\theta_{4}$ are well established and can be obtained from the shapes of constant stress creep curves (Evans [17]). In the current work, these were estimated from experimental creep curves conducted under tension and biaxial stress states in air. The Abaqus implementation for creep included the derived constitutive equation together with a failure criterion connected to critical values of creep continuum damage based on a Kachanov-type damage parameter (Evans [17]). Creep damage is scaled to vary between 0 for virgin material and 1 for an element of material that has failed.

\subsection{Combined creep/fatigue model:}

The model addresses the combined damage accumulation during crack growth through a linear summation of the form: 


$$
\frac{d a}{d N}=\left(\frac{d a}{d N}\right)_{f}+\int\left(\frac{d a}{d t}\right) d t
$$

in which the first term on the RHS represents crack growth due to pure fatigue while the second term provides the additional time dependent contribution due to creep damage. To predict fatigue crack growth rates at high temperatures, the fatigue and creep damage models described above were integrated to form a loosely coupled model with the effects of creep and fatigue calculated separately and then combined at each time increment. The problem with combining the creep model with a fatigue model of this type is that the state variables required to store the creep data $(\mathrm{H}, \mathrm{R}$ and $\mathrm{W}$ variables) are not transferable to subsequent analysis once the crack is incremented to a new length, $\left(\mathrm{a}+\Delta \mathrm{a}_{\mathrm{i}}\right)$ since the nodal coordinates in the component differ between the two meshes. Therefore, an interpolative routine was developed to extrapolate creep damage in the specimen onto the continuously changing fatigue crack mesh (Harrison [20]) method.

A method to solve this problem has been found by storing the creep data for each integration point and the coordinates of those integration points to an external file at the end of each analysis (for each given crack length). During the first iteration of the next analysis this data is read into the subroutine. Then for each integration point of the new analysis, the nearest three old integration points are found using the previously stored coordinates. When the three points have been found their corresponding creep data can be used to calculate the $\mathrm{H}, \mathrm{R}$ and $\mathrm{W}$ values at the new point. An example of this approach can be seen in fig. 4, in which the crack is advanced an arbitrary increment (i.e. $\Delta \mathrm{N}_{\mathrm{i}}$ ) of $0.5 \mathrm{~mm}$ in a $2 \mathrm{D}$ representation of a DEN specimen. This demonstrates how the creep damage, $\mathrm{W}$, remains unchanged after 
crack growth (from fig. $4 \mathrm{a}$ to fig. $4 \mathrm{~b}$ ) thus providing a means to account for prior creep damage during crack propagation modelling. Note that this increment of $0.5 \mathrm{~mm}$ was chosen to demonstrate the approach used and is considerably larger than actual increments used, determined using equation 1 (and in the order of $2-10 \mu \mathrm{m}$, depending on crack length).

For a given crack length, a, the cracked component geometry was held at the maximum load for a period equivalent to the duration of life predicted for the previous increment at crack length $\mathrm{a}-\Delta \mathrm{a}_{(\mathrm{i}-1)}$. This is based on the assumption that creep damage occurs primarily during the one second hold period of the 1-1-1-1 trapezoidal waveform used experimentally. For the starting crack configuration, this time was estimated based on a pure fatigue life prediction (incremental life, $\Delta \mathrm{N}_{1}$ in the absence of creep damage). An elastic-viscoplastic analysis was performed at each increment of crack growth based on the "*Creep" user-subroutine available in Abaqus. The accumulated fraction of creep damage was calculated allowing the proportion of total crack tip life attributable to fatigue damage to be calculated based on equation 12. This value was then used to calculate the number of fatigue cycles, $\Delta \mathrm{N}_{\mathrm{i}}$, required to fail the ligament of material within the crack tip cyclic plastic zone, which is reduced due to creep damage.

\section{Results}

\subsection{Modelling fatigue crack propagation rates at $20^{\circ} \mathrm{C}$}

Figure 5 shows the predicted relationship between crack length and number of applied cycles for the simple case of a crack growing under a simulated fatigue cycle of $\mathrm{R}=$ 0.1 at ambient temperature. Figure 6 shows the resulting stage II fatigue crack 
growth rate prediction plotted against $\Delta \mathrm{K}$. The predictions were obtained using twodimensional plane strain analysis and based on correlation with a Walker strain fatigue damage curve (fig.3a). Also shown in fig.6 are data obtained experimentally from crack growth rate measurements in a corner cracked specimen for this material (Jones et al. [1]). The values of $\Delta \mathrm{K}$ on which both the model and experimental data are plotted were obtained from finite element analysis. Figure 6 illustrates how the model provides a good prediction of the stage II fatigue crack growth rate curve in $\mathrm{Ti}$ 6246 tested in air at ambient temperatures.

\subsection{Modelling creep/fatigue interactions at $500^{\circ} \mathrm{C}$}

Figure 7 shows predicted fatigue crack propagation curves, based on correlation with fig. $3 \mathrm{~b}$, for two different modelling scenarios at $500^{\circ} \mathrm{C}$ in $\mathrm{Ti} 6246$ at $\mathrm{R}=0.1$ as well as experimentally measured crack growth rates (Jones et al. [1]). In the first case, creep processes such as strain accumulation and damage were ignored in the calculation so that crack propagation rates were predicted in the same way as in section 5.1. It is evident from fig. 7 that the model does not provide an adequate prediction of fatigue crack growth rates when creep effects are ignored during calculations. However, when both creep and fatigue damage were considered, using the approach outlined in Section 4.3, the predicted fatigue crack growth rate was considerably higher. In this case, crack growth rate calculations were based on the total accumulated strain after creep (during the aggregate hold period at maximum load for the crack increment) and creep damage calculated using the model described in Section 4. The accuracy of the prediction is highlighted by comparison with the measured cyclic and dwell growth rates. It should be noted that crack growth was simulated in a double-edge notch 
specimen in this case as the method described in Section 4.1 is equally applicable to this specimen geometry.

\section{Discussion}

In this paper, a novel model for predicting fatigue crack growth rates in Ti6246 alloy, based on damage accumulation principles, was presented. The model provides an excellent prediction of the stage II fatigue crack growth rate curve in Ti 6246 alloy tested in air at ambient temperatures. When a creep damage law was integrated into the fatigue model, the observed increase in crack growth rate at elevated temperature was predicted. The combined model represents a significant development in the modelling of fatigue crack propagation at high temperatures and provides the platform for more extensive high temperature fatigue crack growth rate modelling work in subsequent research activities.

Although the prediction of crack growth rate at $500^{\circ} \mathrm{C}$ was found to be very good, it is important to stress that the situation is far more complex than considered in this paper. In the current work, good correlation was achieved with observations when the final value of accumulated strain at each crack micro-increment was used as the basis for damage calculations. Clearly, this is an oversimplification since in reality, stress and strain would be expected to vary with time at high temperatures, leading to a transient stress/strain response in the cyclic plastic zone ahead of a real crack. Environmental effects would also be expected to alter the mechanisms of decohesion during crack growth although crack growth rates experiments performed in hard vacuum at $500^{\circ} \mathrm{C}$ (Jones et al. [1]) clearly demonstrate a contribution of creep to crack propagation rates during dwell loading which strongly suggest that creep/fatigue interactions are 
important in this alloy at $500^{\circ} \mathrm{C}$. However, the simple linear summation of creep/fatigue damage may not be appropriate for the complex stress field within crack tip plastic zone. Therefore, the model should be considered as the first stage towards developing a model capable of predicting fatigue crack growth rates at high temperatures. With the present level of understanding of the complex interactions between the various factors involved (time dependent strain and stress, microstructural damage, environment, etc) it would be very difficult to develop a model that accounts for all these factors. However, this work highlights the importance that creep effects play at high temperatures in Ti 6246 and illustrates how models which rely solely on simulating time-independent plasticity effects whilst ignoring time dependent non-linear effects are deficient.

Experiments confirm that fatigue crack growth rates increase marginally in this alloy with increasing $\mathrm{R}$ ratio (Jones et al. [1]) and one of the critical indicators for the success of any fatigue crack growth rate model is the ability of the method to account for mean stress (i.e. $\mathrm{R}$ ratio) effects. Elsewhere, the model was demonstrated to be insensitive to $\mathrm{R}$ ratio when based on incremental crack steps equivalent to the cyclic plastic zone size (Hurley et al. [8]. This inability to account for load ratio effects in the model is not unexpected given that there are no intrinsic considerations of load ratio in the correlation with strain controlled data given that the empirical Walker strain method removes the $\mathrm{R}$ ratio effects by collapsing the data onto a single $\mathrm{R}=0$ trend-line (Dowling [11]).

A consideration of closure effects in the crack tip region would provide the most obvious explanation for R-ratio dependent crack growth rates in this alloy. Plasticity- 
induced crack closure would lead to residual tensile stresses in the plastic wake causing a reduction in crack growth rate as $R$ value is decreased from $R=0.8$ to $R=$ 0.1. Alternatively, environmental crack closure mechanisms could conceivably account for the load ratio effects observed in this alloy, since oxide formation with thickness comparable to the crack tip opening displacement could lead to reductions in crack propagation rate at lower $\mathrm{R}$ values (Suresh [21]). Closure concepts led to the idea that fatigue crack growth rates should be based on the value of the "effective" stress intensity factor, $\Delta \mathrm{K}_{\mathrm{eff}}$ (Suresh [21]).

In recent years, an alternative "two-parameter" driving force mechanism has been postulated to account for mean stress effects, in which fatigue crack growth rates have been related to both $K_{\max }$ and $\Delta K$ ahead of the crack tip. (Kajawski [22], Sadananda et al. [23], Glinka [24]). This is based on the idea that, within the crack tip process zone, interaction exists between two damage processes, namely monotonic damage due to $\mathrm{K}_{\max }$ and cyclic damage due to $\Delta \mathrm{K}$. It is inferred that the relative contribution to crack growth rate of $\mathrm{K}_{\max }$ increases with increasing load ratio, leading to enhanced crack tip growth rates at higher R-ratios. The present model has been adapted to provide some support for this concept (Hurley et al. [8]). This was done by giving the dimensions of the crack micro-increment, $\Delta \mathrm{a}_{\mathrm{i}}$, a dependency on both $\mathrm{K}_{\max }$ and $\Delta \mathrm{K}$ (i.e. on the peak load and the load range) leading to increasing fatigue crack growth rate predictions with increasing load ratio.

\section{Conclusions}

1. A novel model for predicting fatigue crack growth rates in Ti6246 alloy, based on damage accumulation principles, has been presented. fatigue crack growth is 
assumed to occur as a process of non-continuous incremental steps resulting from damage accumulation in a "process zone" ahead of a propagating fatigue crack.

The size of the process zone, $\Delta \mathrm{a}_{\mathrm{i}}$, was assumed to be equivalent to the width of the cyclic plastic zone.

2. Calculation of $\Delta \mathrm{N}_{\mathrm{i}}$, the number of cycles for crack propagation through the cyclic plastic zone at each stage of incremental crack growth, is equivalent to the fatigue life of a strain controlled specimen in which identical stress-strain conditions apply. The predicted relationship between crack length and applied cycles then provided the basis for calculation of the fatigue crack growth rate, $\Delta \mathrm{a} / \Delta \mathrm{N}$ whilst FE was used to determine $\Delta \mathrm{K}$ as a function of applied cycles. The model provided a good prediction of stage II fatigue crack growth rates in Ti 6246 alloy tested in air at ambient temperatures at $\mathrm{R}=0.1$.

3. To simulate creep at $500^{\circ} \mathrm{C}$, an established creep damage law was implemented to calculate the time dependent stress-strain behaviour and accumulated creep damage ahead of the crack tip for each stage of quasi static crack growth. Higher fatigue crack growth rates have been reported in this material at elevated temperatures and the model successfully demonstrates that fatigue crack growth rates are increased once creep processes are taken into account. Ignoring creep damage and strain accumulation leads to an under-prediction in crack growth rate.

4. At high temperatures, the response of material in the cyclic plastic zone is complex arising from the interaction between the mechanisms of stress relaxation due to creep and damage accumulation due to creep/fatigue. The high temperature creep/fatigue crack growth model presented here should therefore be seen as a first attempt to account for such affects and the paper draws attention to important issues which future models must address. 


\section{Acknowledgments}

The authors would like to express their gratitude to EPSRC and Rolls-Royce plc for financial support. Appreciation is also expressed to Prof. R.W. Evans and Mr W. Harrison for helpful discussions.

\section{References}

[1] Jones J.P., Bache M.R., Evans W.J. and Williams S.R Elevated temperature fatigue crack growth and time dependent behaviour in Ti 6246 The Institute of Materials 2001; 4 (ISSN 1336-5510): 177-185.

[2] Antolovich S.D., Saxena A. and Chanani G.R. Model for fatigue crack propagation. Eng. Fract. Mech. 1975; 7(44): 649-652.

[3] Glinka G. A cumulative model of fatigue crack-growth. Int. J. Fatigue 1982; 4(2): 59-67.

[4] Kujawski D. and Ellyin F. A fatigue crack propagation model. Eng. Fract. Mech. 1984; 20(5/6): 695-704.

[5] Abaqus FE Software Suite, Hibbitt, Karlsson and Sorensen, Pawtucket, RI, USA.

[6] Lemaitre J. and Chaboche J.-L. Mechanics of Solid Materials, Cambridge University Press, UK. 1990.

[7] Hurley P.J., Whittaker M.T., Williams S.R. and Evans W.J. Prediction of fatigue initiation lives in notched Ti 6246 specimens. Int. J. Fatigue (to appear).

[8] Hurley P.J. and Evans W.J. A new model for predicting fatigue crack growth rates. Mat. Sci. Eng. (to appear). 
[9] Pickard A.C. The Application of 3 Dimensional Finite Element Methods to Fracture Mechanics and Fatigue Life Prediction. Chameleon Press Ltd: London, UK. 1986.

[10] Walker K. Effects of Environment and Complex Load History on Fatigue Life, ASTM STP 1970; 462: 1-14.

[11] Dowling N.E. Mechanical Behaviour of Materials ( ${ }^{\text {nd }}$ Edition), Prentice Hall, New Jersey, USA, 1999.

[12] Boyle J.T. and Spence J. Stress analysis for Creep, Butterworths, London, 1983.

[13] Hayhurst D.R and Henderson J.T Creep stress redistribution in notched bars, Int. J. Mech. Sci. 1977; 19(3): 133-146.

[14] Al-Faddagh K.D, Fenner R.T and Webster G. A. Steady state stress distributions in circumferentially notched bars subjected to creep. J.Strain Analysis for Engineering Design (1982); 17(3): 123-132.

[15] Hayhurst D.R., Dimmer P.R. and Morrison C.J. Development of continuum damage in the creep-rupture of notched bars. Phil Trans.R. Soc. Lond. 1984; A311 (1516); 103-129: 1984.

[16] Othman A.M., Hayhurst D.R., and Dyson B.F., Skeletal point stresses in circumferentially loaded tension bars undergoing tertiary creep modelled with physically-based constitutive equations. Proc. R. Soc. Lond., 1993; A441 (1912): 343358.

[17] Evans R.W. A constitutive model for the high-temperature creep of particlehardened alloys based on the $\theta$ projection method. Proc. R. Soc. Lond. A 2000: 835868. 
[18] Zienkiewicz O.C. and Cormeau I. Visco-plasticity - plasticity and creep in elastic solids. A unified numerical solution procedure. Int. J. Num. Meth. Engng. 1974; 8: $821-845$

[19] Evans R.W. and Wilshire B. Introduction to Creep, The Institute of Materials, UK, 1993

[20] Harrison W. Creep Modelling of Ti6246 and Waspaloy using Abaqus. Ph.D thesis, University of Wales Swansea, UK. 2006.

[21] Suresh S. Fatigue of Materials, Cambridge, UK: Cambridge University Press, 1998.

[22] Kajawski D., A new $\left(\Delta K+K_{\max }\right)^{0.5}$ driving force parameter for crack growth in aluminum alloys. Int. J. Fatigue 2001; 23(8): 733-740.

[23] Sadananda, K., Vasudevan, A.K. and Kang, I.W., Effect of superimposed monotonic fracture modes on the $\Delta \mathrm{K}$ and $\mathrm{K}_{\max }$ parameters of fatigue crack propagation. Acta Mater. 2003; 51(12): 3399-3414.

[24] Noroozi A.H., Glinka G. and Lambert S. A two parameter model for fatigue crack growth analysis. Int. J. Fatigue 2005; 27(10-12): 1277-1296. 


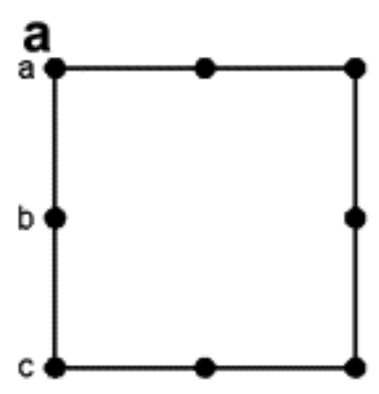

b

$a, b$,

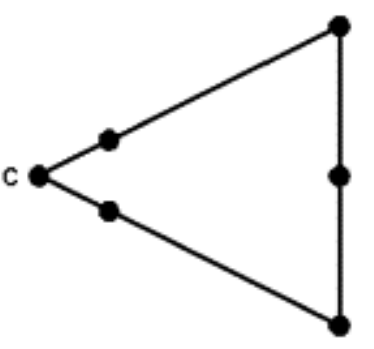

Figure 1: Schematic representation of (a) isoparametric quadratic element with midpoint nodes; and (b) quarter-point element with nodes collapsed to a single point at the crack tip (Abaqus [8]).
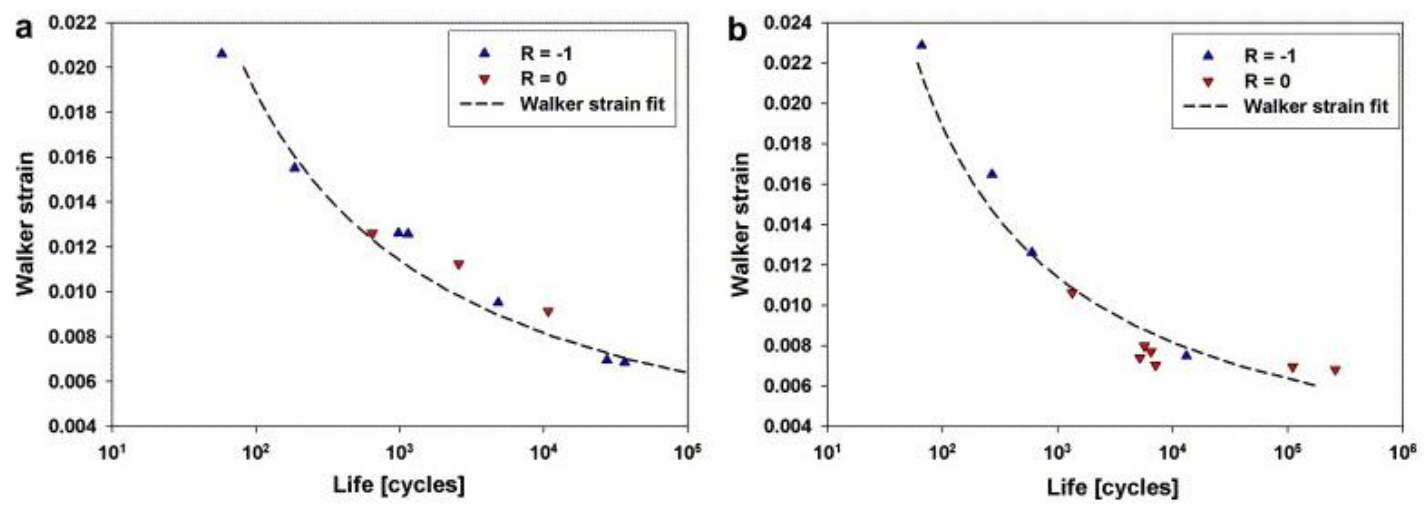

Figure 2: Optimised Walker strain lifing curve for SC specimens tested at (a) $20^{\circ} \mathrm{C}$; and (b) $500^{\circ} \mathrm{C}$. 

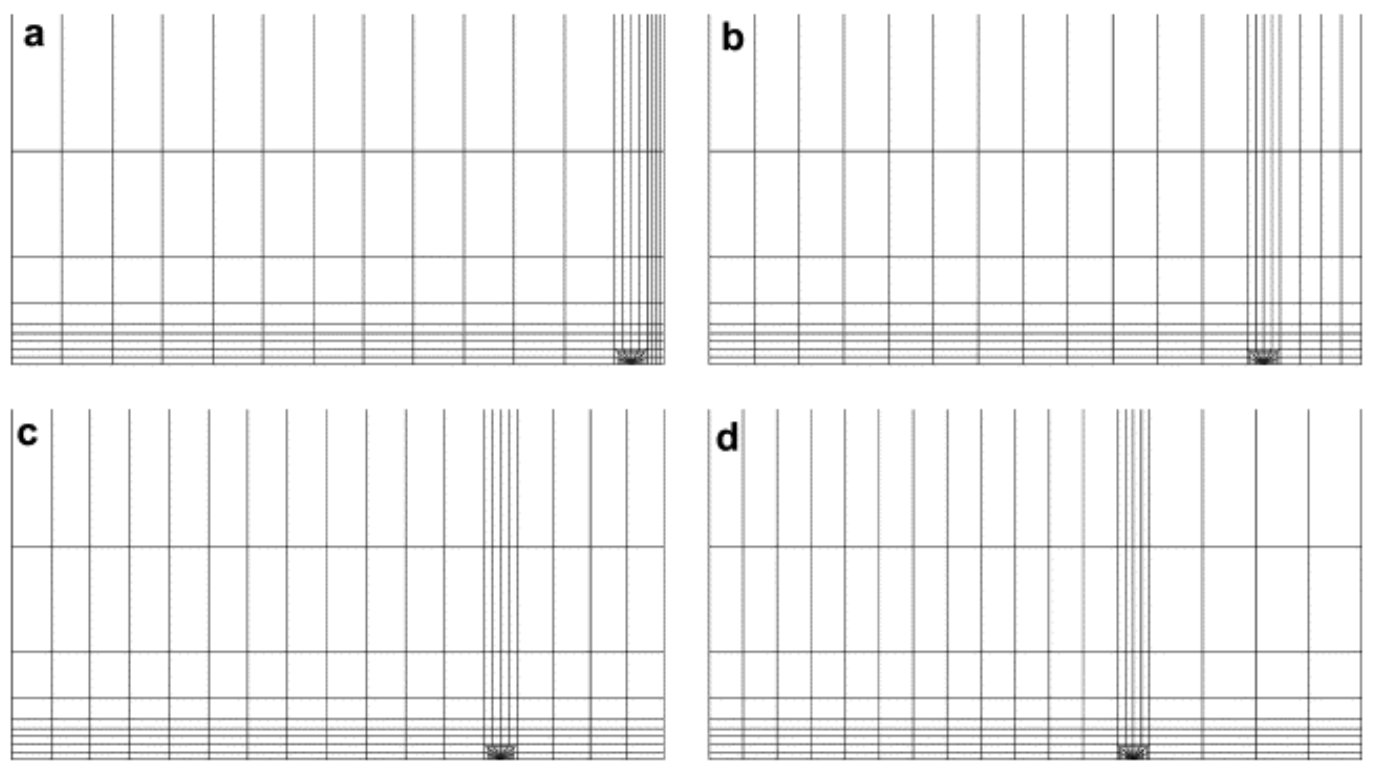

Figure 3: Mesh configuration of a simulated corner cracked specimen at various stages of incremental crack growth with crack length of (a) $0.5 \mathrm{~mm}$; (b) $1.5 \mathrm{~mm}$; (c) $2.5 \mathrm{~mm}$; (d) $3.5 \mathrm{~mm}$. Note that the upper part of the specimen mesh is not shown. 


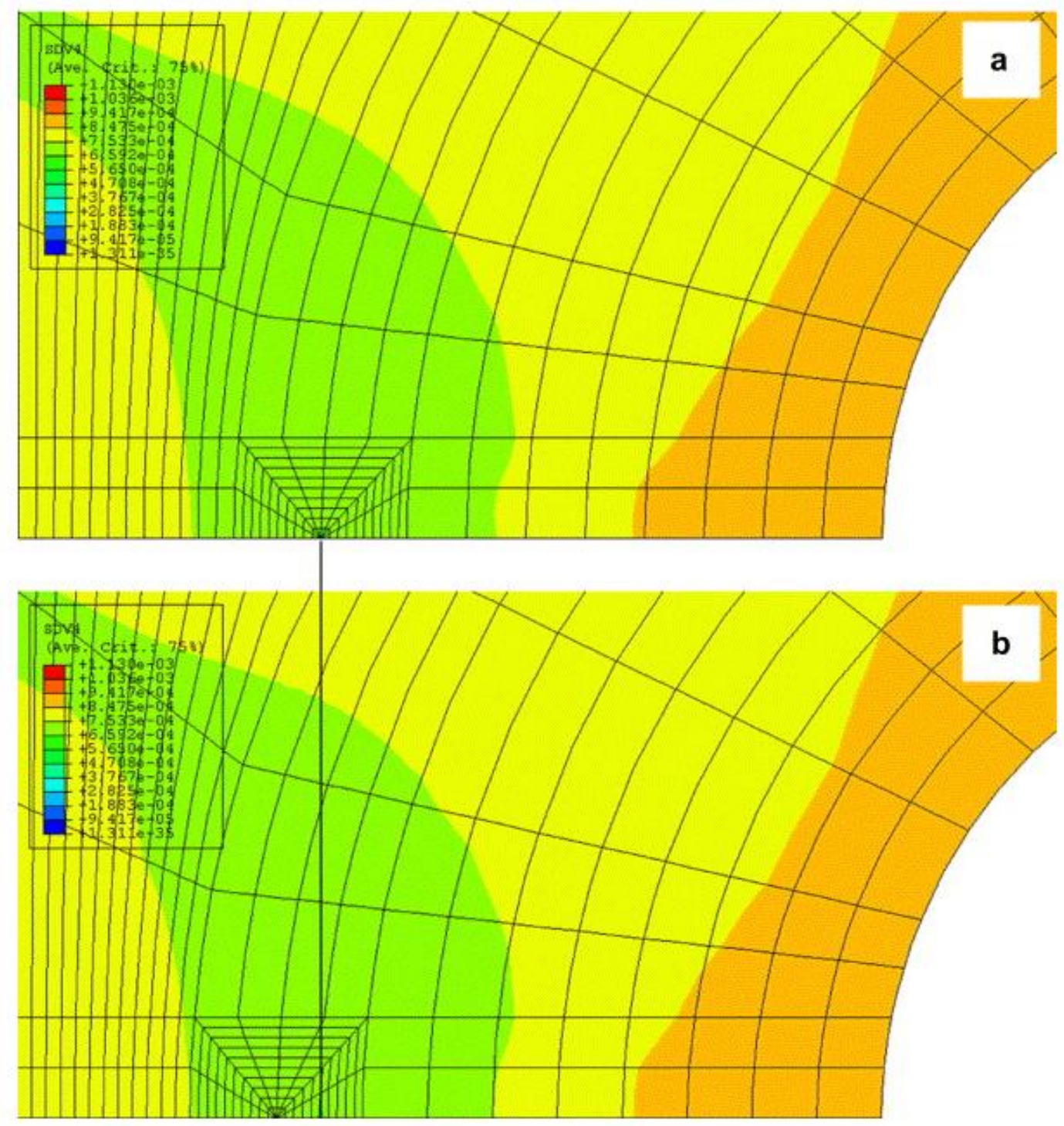

Figure 4: (a) Contour plot of creep damage in a $2 d$ section of a double-edge notch (DEN) specimen with a crack present; and (b) after an arbitrary crack increment of $0.5 \mathrm{~mm}$. This demonstrates how the creep damage function in the specimen can be retained after remeshing to a new crack size (courtesy of W.Harrison). 


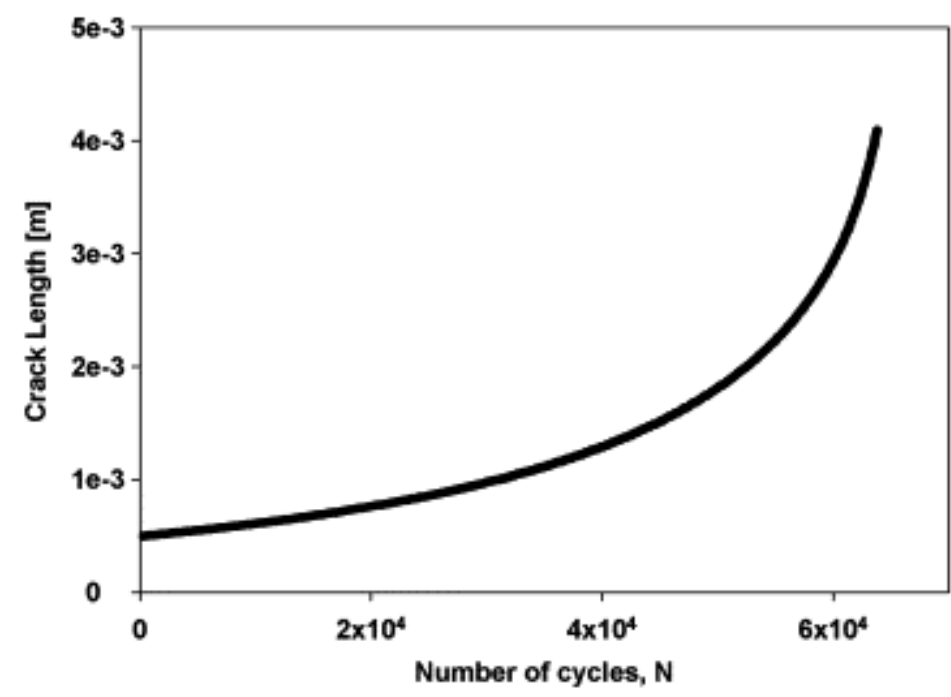

Figure 5: Predicted relationship between number of applied cycles and crack length.

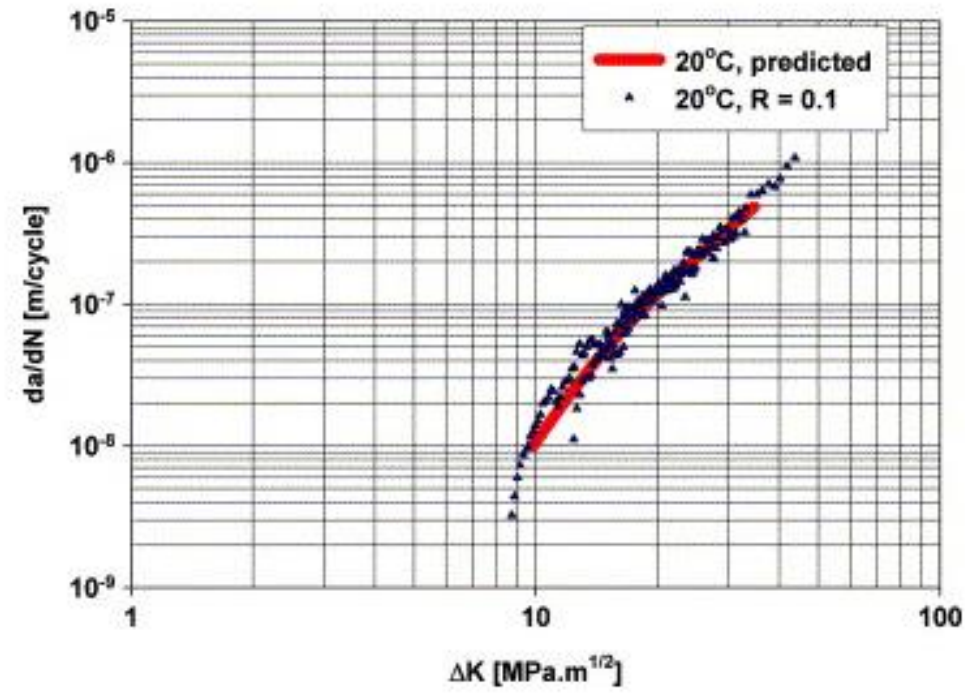

Figure 6: Measured (Jones et al. [1]) and predicted crack fatigue crack growth rates in Ti 6246 corner cracked specimens at $20^{\circ} \mathrm{C}$. 


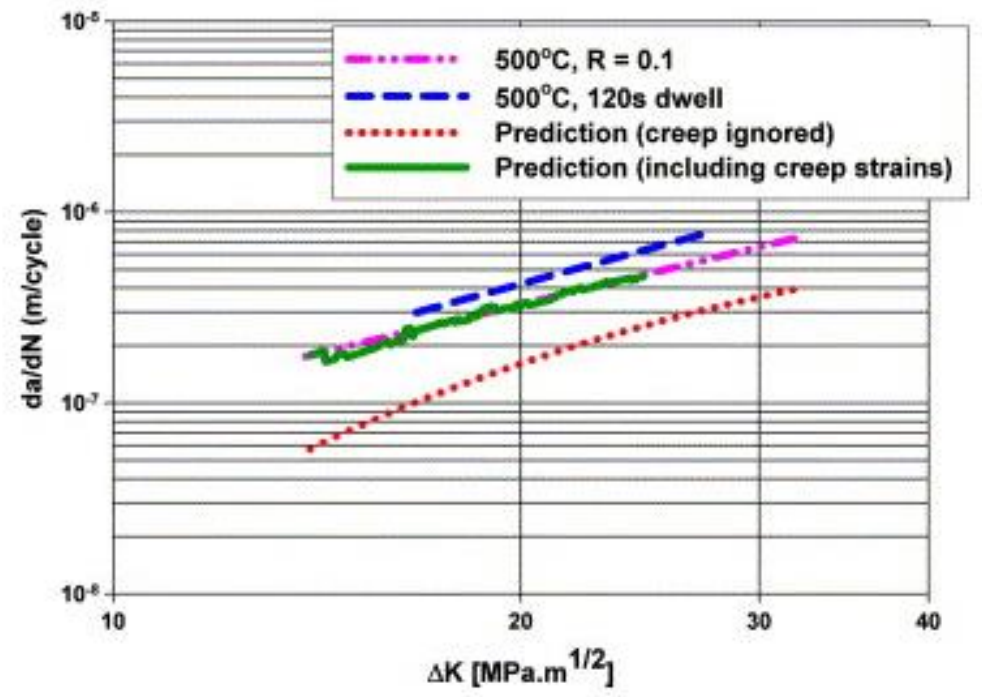

Figure 7: Measured (Jones et al. [1]) and predicted crack fatigue crack growth rates in Ti 6246 DEN specimens at $500^{\circ} \mathrm{C}$. Two modelling scenarios were considered: (i) the model ignores creep processes (dotted line); and (ii) the model accounts for creep damage and total accumulated strain after creep (solid line). 